\title{
miR-629 targets FOXO3 to promote cell apoptosis in gastric cancer
}

\author{
MING LI ${ }^{1,2}$, YINGXIN WANG ${ }^{2}$, XIA LIU $^{3}$, ZHENDUO ZHANG ${ }^{2}$, LIWEI WANG ${ }^{2}$ and YONG LI $^{4}$ \\ ${ }^{1}$ Department of General Surgery, Hebei Medical University, Shijiazhuang, Hebei 050017; \\ ${ }^{2}$ Department of General Surgery, The First Hospital of Shijiazhuang City, Shijiazhuang, Hebei 050011; \\ ${ }^{3}$ Department of Forensic Pathology, Hebei Medical University, Shijiazhuang, Hebei 050017; \\ ${ }^{4}$ Department of General Surgery, Fourth Hospital of Hebei Medical University, Shijiazhuang, Hebei 050011, P.R. China
}

Received June 27, 2018; Accepted November 1, 2019

DOI: $10.3892 / \mathrm{etm} .2019 .8168$

\begin{abstract}
Gastric cancer (GC) is one of the most aggressive types of human tumor worldwide, and the 5-year survival rate is less than $25 \%$. The transcriptional factor, forkhead box $\mathrm{O} 3$ (FOXO3), is regulated by various micro (mi)RNAs and has been reported to be associated with multiple regulatory signaling pathways involved in tumor development. The current study therefore assessed the impact of miR-629 and FOXO3 on gastric cancer. Reverse transcription-quantitative polymerase chain reaction and western blotting were performed to assess the expression of mRNA and protein, respectively. Additionally, the cell proliferation and apoptosis rate were determined via an MTT assay and flow cytometry, respectively. The online database TargetScan predicted that FOXO3 was a target of miR-629. A luciferase reporter assay was also performed to verify that FOXO3 was the direct target of miR-629. The results demonstrated that miR-629 and FOXO3 was upregulated and downregulated in GC tissue, respectively. Furthermore, following transfection with a miR-629 inhibitor, SGC-7901, cell proliferation and apoptosis rate were inhibited and promoted when compared with the control group, respectively. Moreover, after the treatment with SGC-7901, the expression of FOXO3, Bax, Caspase 3 was upregulated, and Bcl-2 was downregulated. Furthermore, the luciferase reporter assay revealed that FOXO3 was the target of miR-629. The results demonstrated that miR-629 and FOXO3 serve vital roles in the development of gastric cancer and may be a future therapeutic target.
\end{abstract}

Correspondence to: Dr Yong Li, Department of General Surgery, Fourth Hospital of Hebei Medical University, 12 Jiankang Road, Shijiazhuang, Hebei 050011, P.R. China

E-mail: dr_yongli@aliyun.com

Key words: forkhead box O3, miRNA-629, apoptosis, gastric cancer, proliferation

\section{Introduction}

Gastric cancer (GC) is one of the leading causes of mortality worldwide, particularly in Asian countries such as China (1). Over the past three decades, the incidence of gastric cancer has gradually declined due to improved treatment (2). However, the mortality rate of gastric cancer remains high and its burden on the patients remains substantial $(3,4)$. It was reported that the 5 year survival rate of patients with GC treated at an early stage was $90-95 \%$ in Japan from 1970 to 2000; however, the overall survival rate of late stage GC is less than $20 \%(5,6)$. Despite advances in GC treatment, a substantial number of patients exhibit local recurrence or distant metastasis, the underlying molecular mechanism of which remains unclear (7). Cisplatin and fluoropyrimidine-based chemotherapy, in combination with trastuzumab, is widely used to treat patients with stage IV GC that are human epidermal growth factor receptor 2-positive and who are eligible to receive chemotherapy (8). Recently, it has been demonstrated that biological therapies may be novel treatments of GC (9). For instance, overexpressed miR-200b inhibited the proliferation and migration of GC cells (10). The current study therefore aimed to assess the impact of micro (mi)RNAs (miRs) on the development of GC.

miRNAs are a group of small non-coding RNAs that are comprised of approximately 22-24 nucleotides (11). miRNAs specifically bind to the $3^{\prime}$ untranslated region (3'-UTR) of target mRNAs and as a result, promote mRNA degradation and alter protein expression (12). In addition, miRNA expression profiles in certain types of cancer serve primary roles in various human biological processes, including migration, cellular metabolism, cell proliferation, apoptosis and epithelial-mesenchymal transition (13). Previous studies have revealed that the dysregulation of certain miRNAs in Helicobacter pylori-infected gastric mucosa and GC, including miR-17-5p/20a, miR-106b and miR-93, induce the development and progression of cancer, involving cell proliferation, migration, invasion and apoptosis (14-17). Furthermore, other findings have indicated that miR-627, miR-629 and miR-652 are highly expressed in patients with GC (7). In addition, human pancreatic cancer progression was induced by miR-629 via FOXO3 targeting (18).

Forkhead box O3 (FOXO3) is a transcription factor belonging to the FOXO family, the effects of which have been 
assessed in a wide variety of cellular processes, including proliferation, cell cycle arrest, cell death and metabolism (19,20). A previous study has indicated that FOXO3A promoted GC cell migration and invasion via the induction of cathepsin L (21). Additionally, FOXO3 exhibits tumor suppressive effects on $\mathrm{GC}$, which might be a promising therapy in clinic (22). However, the impact of miR-629 on FOXO3 in GC has not yet been fully elucidated. Based on previous studies $(18,21)$, the current study hypothesized that miR-629 may target FOXO3 to affect GC cell activity.

The expression of miR-629 and FOXO3 in GC, and the impact of miR-629 on FOXO3 expression and associated cell activity, including cell proliferation and cell apoptosis, were assessed in the current study. The results demonstrated that miR-629 may serve as a therapeutic target for GC and that the effects of miR-629 indicate that it may serve as a novel molecular target for GC diagnosis and treatment.

\section{Materials and methods}

Patients and tissues. A total of 44 patients with GC recruited into the present study were hospitalized in The First Hospital of Shijiazhuang City (Shijiazhuang City, China) from June 2016 to August 2017. The patients (15 females and 29 males) aged between 36 and 67 years old. None of the patients had undergone surgical treatment, radiotherapy or chemotherapy prior to enrollment. Tumor and paracarcinoma tissues (obtained from each patient $5 \mathrm{~cm}$ away from the edge of tumor) were stored in $10 \%$ formalin at room temperature. The paracarcinoma tissues were used as the control group. The current study was approved by the Ethics Committee of The First Hospital of Shijiazhuang City and all patients provided their written informed consent.

Cell line and plasmids. The SGC-7901 cell line was purchased from the American Type Culture Collection (Manassas, VA, USA) and maintained in RPMI-1640 medium containing $10 \%$ fetal bovine serum (both Thermo Fisher Scientific, Inc., Waltham, MA, USA). All cells were cultured in a humidified incubator at $37^{\circ} \mathrm{C}$ in $5 \% \mathrm{CO}_{2}$ for $24 \mathrm{~h}$. The miR-629 inhibitor (5'-GCUGGGCUUACGUUGGAGAAC-3'), negative control (5'-TAACACGTCTATACGCCCA-3'), FOXO3 3'UTR wild-type (WT) and FOXO3 3'UTR-mutant (MUT) were constructed by OriGene Technologies, Inc. (Rockville, MD, USA).

miRNA transfection. SGC-7901 cells were seeded in 12-well plates $\left(2 \times 10^{5}\right.$ cells/well) with RPMI-1640 medium and incubated at $37^{\circ} \mathrm{C}$ with $5 \% \mathrm{CO}_{2}$. Once cells reached a confluence of $60-70 \%$, transfection was performed. miR-629 inhibitor or miR-NC inhibitor (50 nmol/l) was transfected into cells using Lipofectamine ${ }^{\circledR} 2000$ (Thermo Fisher Scientific, Inc.) for $6 \mathrm{~h}$ according to the manufacturer's protocol. Subsequently, transfected cells were cultured in RPMI-1640 medium containing $10 \%$ fetal bovine serum in a humidified incubator at $37^{\circ} \mathrm{C}$ with $5 \% \mathrm{CO}_{2}$ for $48 \mathrm{~h}$. At 48 -h post transfection, cells were used for the subsequent experiments.

Reverse transcription-quantitative polymerase chain reaction $(R T-q P C R)$. RNA was extracted from GC and paracarcinoma tissues, and SGC-7901 cells using the TRIzol ${ }^{\circledR}$ reagent (Invitrogen; Thermo Fisher Scientific, Inc.). Subsequently, RNA concentration was determined using Nanodrop 2000 (Thermo Fisher Scientific, Inc.). Takara Taq polymerase (Takara Bio, Inc., Otsu, Japan) was used for DNA amplification. cDNA was synthesized using the PrimeScript RT reagent kit (Takara Biotechnology, Co., Ltd., Dalian, China) at $37^{\circ} \mathrm{C}$ for 15 min. qPCR was performed using the SYBR Premix EX Taq ${ }^{\mathrm{TM}}$ kit (Takara Bio, Inc.). The thermocycling conditions were as follows: Initial denaturation at $95^{\circ} \mathrm{C}$ for $30 \mathrm{sec}$, followed by 40 cycles at $95^{\circ} \mathrm{C}$ for $30 \mathrm{sec}, 60^{\circ} \mathrm{C}$ for $30 \mathrm{sec}$ and $72^{\circ} \mathrm{C}$ for $30 \mathrm{sec}$, and then a final extension at $72^{\circ} \mathrm{C}$ for another $10 \mathrm{~min}$. The expression of miR-629 was normalized to the endogenous expression of U6 and the relative expression of protein mRNA was normalized to GAPDH. The $2^{-\Delta \Delta \mathrm{Cq}}$ method was used to analyze mRNA expression (23). The sequences of the primers were listed in Table I.

Western blotting. Western blot analysis was performed to assess protein expression. Transfected SGC-7901 cells were lysed using radioimmunoprecipitation assay buffer (cat. no. P0013B; Beyotime Institute of Biotechnology, Haimen, China) and centrifuged at $10,000 \mathrm{x}$ g for $15 \mathrm{~min}$ at $4^{\circ} \mathrm{C}$. Protein concentration was then determined using a BCA kit (Thermo Fisher Scientific, Inc.). Subsequently, SDS-PAGE containing $50 \mathrm{mg}$ protein/lane was performed with $25 \%$ resolving gel. Proteins were transferred onto polyvinylidene difluoride membranes, which were blocked with $15 \%$ skimmed milk for $1 \mathrm{~h}$ at $37^{\circ} \mathrm{C}$ prior to experimentation. GAPDH was utilized as an internal control. Then the membranes were incubated with primary anti-FOXO3 (cat no. ab109629; 1:1,000), anti-Bcl-2 associated x (Bax; cat no. ab32503; 1:1,000), anti-B-cell lymphoma 2 (Bcl-2; cat no. ab32124; 1:1,000), anti-caspase-3 (cat no. ab32351; 1:5,000) and anti-GAPDH (cat no. EPR16891; 1:10,000; all Abcam, Cambridge, MA, USA) and horseradish peroxidase-conjugated secondary antibodies (cat no. ab6721; 1:5,000; Abcam) overnight at $4^{\circ} \mathrm{C}$ and incubated at room temperature for $1 \mathrm{~h}$. An ECL kit (cat. no. K820500; Biovision Inc., Milpitas, CA, USA) was used to visualize samples. The gray value was obtained using ImageJ analysis software (National Institutes of Health, Bethesda, MD, USA).

MTT assay. At $48 \mathrm{~h}$ following plasmid transfection, an MTT assay was performed to assess the viability of SGC-7901. Cells were seeded into 96 -well plate $\left(5 \times 10^{3}\right.$ cells/well). Purple formazan was dissolved using DMSO and optical density was then measured at $595 \mathrm{~nm}$ using a microplate reader at 0,12 , 24 and $48 \mathrm{~h}$.

Flow cytometry assay. Following $48 \mathrm{~h}$ incubation, SGC-7901 cells were digested with trypsin for $2 \mathrm{~min}$ at $37^{\circ} \mathrm{C}$. The apoptosis rate of GC cells was determined with an Annexin V-FITC Apoptosis Detection kit (Abcam) according to the manufacturer's protocol. Cells were stained with $5 \mu \mathrm{l}$ Annexin V and propidium iodide (BD Bioscience, Franklin Lakes, NJ, USA) in shade at $37^{\circ} \mathrm{C}$ for $15 \mathrm{~min}$ prior to counting. The apoptosis rate of transfected SGC-7901 cells was then counted using a flow cytometer (EPICS XL; Beckman Coulter, Inc., Fullerton, CA, USA) with FCS Express 3.0 software (DeNovo Software, Glendale, CA, USA). 
Table I. Oligonucleotide primers used reverse transcriptionquantitative polymerase chain reaction analysis.

\begin{tabular}{lcl}
\hline Gene & Direction & \multicolumn{1}{c}{ Sequence (5'-3') } \\
\hline miR-629 & $\mathrm{F}$ & TGGGTTTACGTTGGGAGA \\
& $\mathrm{R}$ & GTGCAGGGTCCGAGGTATTC \\
U6 & $\mathrm{F}$ & CTCGCTTCGGCAGCACA \\
& $\mathrm{R}$ & AACGCTTCACGAATTTGCG \\
Bax & $\mathrm{F}$ & CACCAGCTCTGAACAGATCATGA \\
& $\mathrm{R}$ & TCAGCCCATCTTCTTCCAGATGT \\
Bcl-2 & $\mathrm{F}$ & CACCCCTGGCATCTTCTCCTT \\
& $\mathrm{R}$ & AGCGTCTTCAGAGACAGCCAG \\
Caspase-3 & $\mathrm{F}$ & GATGTGGACGCAGCCAACCTCA \\
& $\mathrm{R}$ & TCCGGCAGTAGTCGCCTCTGAA \\
FOXO3 & $\mathrm{F}$ & TCACGCACCAATTCTAACGC \\
& $\mathrm{R}$ & CACGGCTTGCTTACTGAAGG \\
GAPDH & $\mathrm{F}$ & AGAAGGCTGGGGCTCATTTG \\
& $\mathrm{R}$ & GCAGGAGGCATTGCTGATGAT
\end{tabular}

miR, microRNA; Bax, Bcl-2 associated x; Bcl-2, B-cell lymphoma 2; FOXO3, forkhead box O3; F, forward; R, reverse.

Bioinformatics analysis and the dual-luciferase-reporter assay. miR-629 target gene prediction was determined using TargetScan (http://www.targetscan.org/vert_71/). For the luciferase reporter assay. The pMIR-REPORT ${ }^{\mathrm{TM}}$ Luciferase plasmid was purchased from Thermo Fisher Scientific, Inc. SGC-7901 cells $\left(5 \times 10^{3}\right)$ were seeded into 96-well plates and incubated with RPMI- 1640 medium at $37^{\circ} \mathrm{C}$ with $5 \% \mathrm{CO}_{2}$ until a confluence of $70 \%$ was reached. Subsequently, the miR-629 inhibitor and negative control plasmid with 3'UTR-FOXO3-WT and 3'UTR-FOXO3-MUT were transfected into SGC-7901 cells using Lipofectamine ${ }^{\circledR} 3000$ (Thermo Fisher Scientific, Inc.) and incubated at $37^{\circ} \mathrm{C}$. After $48 \mathrm{~h}$, the dual-luciferase reporter assay kit (Promega Corporation, Madison, WI, USA) was utilized for luciferase determination and luciferase activity was normalized to that of Renilla.

Statistical analysis. All data were presented as the mean \pm standard deviation and were performed in triplicate. A Student's t-test was performed for comparisons between two groups and one-way analysis of variance followed by a Newman-Keuls post-hoc test was utilized for the comparison of multiple groups. Analysis was performed using GraphPad Prism version 5.01 software (GraphPad Software, Inc., La Jolla, CA, USA). $\mathrm{P}<0.05$ was considered to indicate a statistically significant difference.

\section{Results}

FOXO3 is downregulated but miR-629 is upregulated in GC tissues. The expression of miR-629 and FOXO3 mRNA was determined using RT-qPCR and FOXO3 protein expression was assessed via western blot analysis. As shown in Fig. 1A, the results demonstrated that FOXO3 is significantly downregulated in GC tumor tissues at the mRNA and protein level when compared with the control group. By contrast, significant upregulation in miR-629 was observed in tumor tissues compared with control tissues (Fig. 1B).

miR-629 is downregulated in SGC-7901 cells following inhibitor transfection. Cells were divided into three groups: The blank control group, the miR-NC inhibitor group and the miR-629 inhibitor group. As shown in Fig. 2, miR-629 expression was significantly decreased in the inhibitor group compared with the control. These results indicate that the miR-629 inhibitor was successfully constructed and thus could be utilized for further experimentation.

$S G C-7901$ cell proliferation rate is inhibited by the miR-629 inhibitor. The proliferation rate of SGC-7901 cells was assessed via an MTT assay. As presented in Fig. 3, compared with the control group, the proliferation rate significantly decreased in the inhibitor group following 24 and $48 \mathrm{~h}$. Thus, the results demonstrated that the miR-629 inhibitor induces a decrease in SGC-7901 cell proliferation.

SGC-7901 cell apoptosis is promoted by the miR-629 inhibitor. SGC-7901 cell apoptosis was assessed using flow cytometry. The results indicated that the cell apoptosis rate was significantly increased in the inhibitor group compared with the control group (Fig. 4A and B). It was therefore concluded that miR-629 inhibits SGC-7901 cell apoptosis.

FOXO3, Bax and caspase-3 are upregulated but Bcl-2 is downregulated in SGC-7901 cells following inhibitor transfection. The expression of FOXO3, Bax, caspase-3 and Bcl-2 in SGC-7901 cells was assessed following miR-629 inhibitor transfection. The results demonstrated that the expression of FOXO3, Bax and caspase-3 mRNA was significantly increased in the inhibitor group compared with the control group. Additionally, Bcl-2 expression was significantly decreased in the inhibitor group compared with the control group (Fig. 5A-D). Furthermore, western blot analysis revealed that protein expression was consistent with that of mRNA (Fig. 5E and F). These results indicate that the miR-629 inhibitor downregulates $\mathrm{Bcl}-2$ and upregulates the expression of FOXO3, Bax and caspase-3.

Luciferase reporter assay for target verification. TargetScan was utilized to predict the target of miR-629. The results revealed that $\mathrm{FOXO} 3$ was a target of miR-629 due to binding at the 3'UTR of FOXO3 (Fig. 6A). The luciferase activity significantly reduced in the FOXO3 3'UTR-WT group compared with the negative control group. Furthermore, there was no significant difference between the negative control group and the FOXO3 3'UTR-MUT group (Fig. 6B). These results indicate that FOXO3 is the direct target of miR-629.

\section{Discussion}

In congruence with a study by Shin et al (7) the present study demonstrated that miR-629 is overexpressed and FOXO3 is significantly downregualted in GC. Furthermore, a study by Xiong et al (24) revealed that sphingosine kinase 1 induces the phosphorylation of FOXO3 and subsequent downregulation 
A

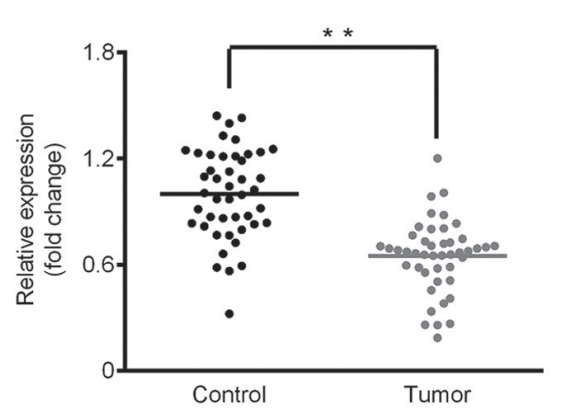

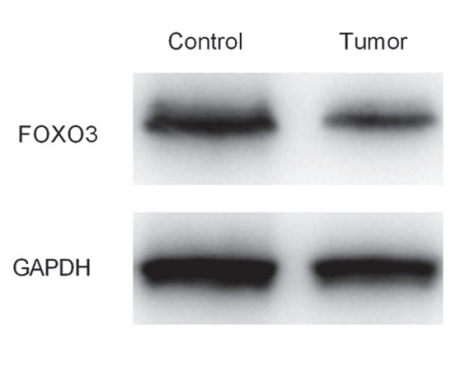

B



Figure 1. Downregulated FOXO3 and upregulated miR-629 expression in tissues with gastric cancer. (A) FOXO3 mRNA and protein expression were determined using RT-qPCR and western blotting, respectively. A sample from one patient is presented. (B) miR-629 expression was determined using RT-qPCR. ${ }^{* *} \mathrm{P}<0.01$. FOXO3, forkhead box O3; miR, miRNA; control, paracarcinoma tissues; tumor, tumor tissues.

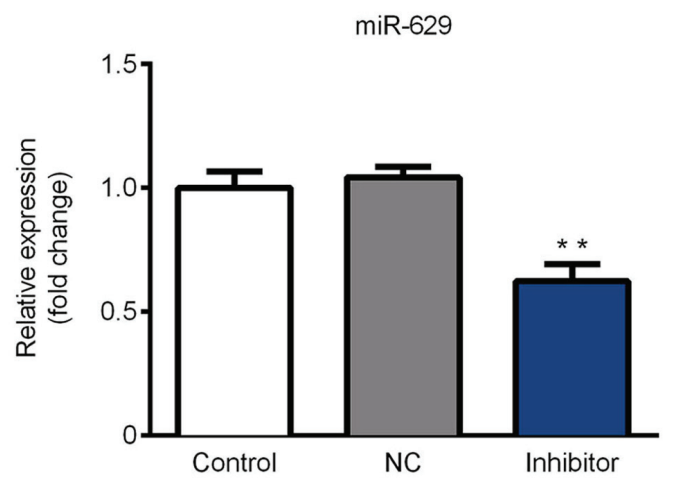

Figure 2. miR-629 is downregulated in SGC-7901 cells following inhibitor transfection. The expression of miR-629 was determined via reverse transcription-quantitative polymerase chain reaction. ${ }^{* *} \mathrm{P}<0.01 \mathrm{vs}$. the control group. miR, microRNA; control, non-transfected group; NC, transfected with negative control plasmids; inhibitor, transfected with miR-629 inhibitor plasmids.

of translational activity, resulting in phosphoinositide 3-kinase/protein kinase B signaling. Further studies should therefore assess the factors that affect the expression of FOXO3 in GC. The results of the current study revealed that miR-629 binds to FOXO3 and that the decreased expression of the latter may be induced by GC. It has been demonstrated that miR-629 promotes the progression of human pancreatic cancer by targeting FOXO3, resulting in enhanced pancreatic carcinoma cell proliferation and invasion (18). The present study also demonstrated that downregulated miR-629 suppressed SGC-7901 cell proliferation.

FOXO3 transcription factors are an evolutionarily conserved subfamily of the forkhead transcription factors, which are characterized by a forkhead DNA-binding domain (25). The FOXO subfamily comprises FOXO1, FOXO3, FOXO4 and FOXO6 (26). Furthermore, the mediation of these transcription factors is determined by the availability of certain growth factors, such as insulin and tumor necrosis factor $\alpha(26,27)$. In addition, it has been revealed that FOXO transcription factors exert regulatory effects on cell growth, the cell cycle, apoptosis and defense against oxidative stress (28). The current study demonstrated that the expression of FOXO3, Bax and caspase-3 was upregulated and that $\mathrm{Bcl}-2$ expression was suppressed, which was consistent with the apoptosis results.

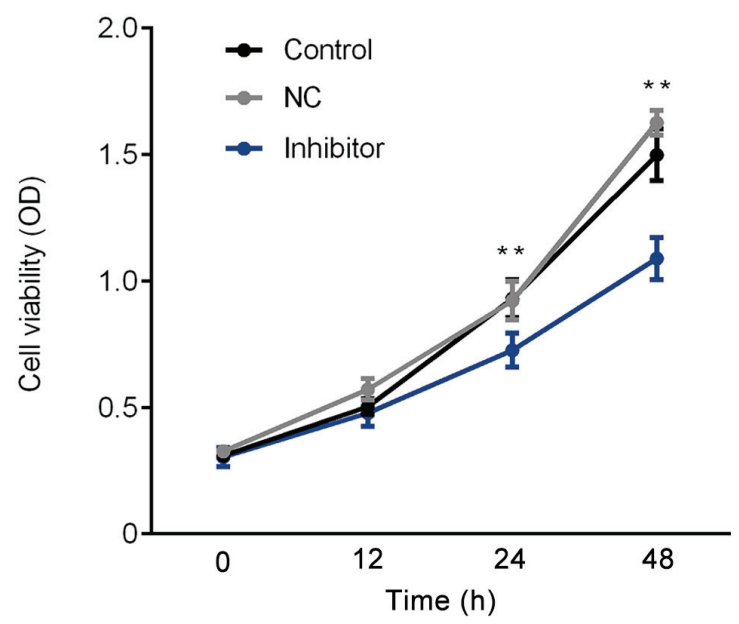

Figure 3. SGC-7901 cell proliferation rate is inhibited following transfection with a miR-629 inhibitor. The proliferation of SGC-7901 cells was determined via an MTT assay. ${ }^{* *} \mathrm{P}<0.01$ vs. the control group. miR, microRNA; control, non-transfected group; $\mathrm{NC}$, transfected with negative control plasmids; inhibitor, transfected with miR-629 inhibitor plasmids; OD, optical density.

These results indicate that suppressed miR-629 upregulated the expression of FOXO3, and promoted cell apoptosis by reducing the expression of Bcl-2, and increasing BAX and Caspase 3 expression. A recent study has revealed that cyclin-dependent kinase 6 protects against epithelial ovarian cancer by regulating FOXO3 and promoting cell death (29).

The present study also revealed that miR-629 targets FOXO3, resulting in the reduced expression of FOXO3 and the progression of GC. The results further demonstrated that the miR-629 inhibitor reduced cell proliferation and promoted cell apoptosis. The expression of apoptosis-associated proteins was also assessed and the results of western blotting indicated that cell apoptosis activity had increased. Furthermore, the current study revealed that miR-629 binds to the 3'UTR Of FOXO3, indicating that it is a target of miR-629. Studies have demonstrated that various miRNAs, including miR-223, miR-122 and miR-451 are associated with the progression, migration and invasion of GC cell $(30,31)$. However, miR-223 also enhanced chemosensitivity and promoted the apoptosis of GC cells by targeting FOXO3 (32). Additionally, TargetScan has revealed that FOXO3 is the target of miR-629. Pancreatic 
A

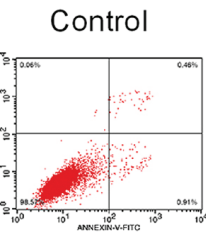

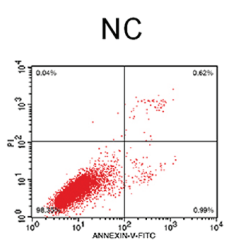

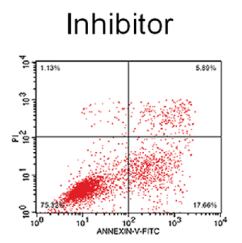

B



Figure 4. SGC-7901 cell apoptosis is promoted following transfection with the miR-629 inhibitor. Flow cytometry was utilized to assess SGC-7901 cell apoptosis. (A) Flow cytometric scatter plots and (B) quantification of apoptosis rate. ${ }^{* *} \mathrm{P}<0.01$ vs. the control group. miR, microRNA; control, non-transfected group; $\mathrm{NC}$, transfected with negative control plasmids; inhibitor, transfected with miR-629 inhibitor plasmids; PI, propidium iodide; FITC, fluorescein isothiocyanate.

A

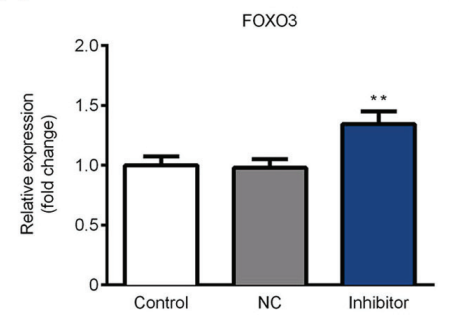

D

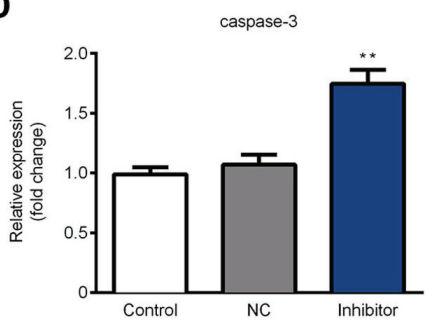

B

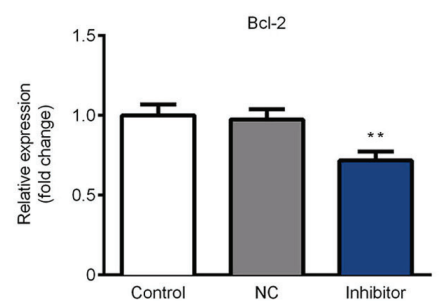

E

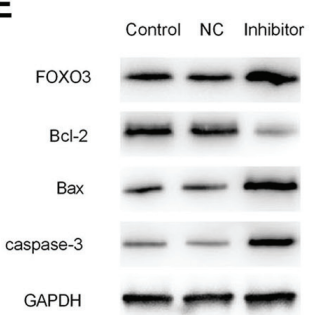

C

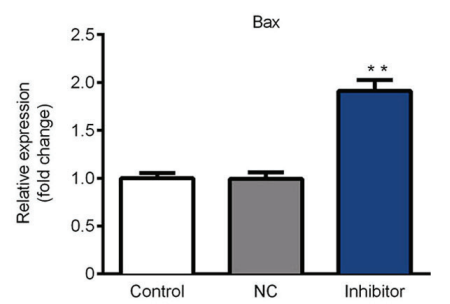

$\mathbf{F}$



Figure 5. miR-629 inhibitor transfection upregulates FOXO3, Bax and caspase-3, and downregulates Bcl-2 in SGC-7901 cells. The expression of (A) FOXO3, (B) Bax, (C) Bcl-2 and (D) caspase-3 mRNA was determined using a reverse transcription-quantitative polymerase chain reaction assay. (E) The protein level of FOXO3, Bax, Bcl-2 and caspase-3 was determined using western blotting. (F) Quantification of western blotting results. ${ }^{* *} \mathrm{P}<0.01$ vs. the control group miR, microRNA; FOXO3, forkhead box O3; Bax, Bcl-2 associated x; Bcl-2, B-cell lymphoma 2; control, non-transfected group; NC, transfected with negative control plasmids; inhibitor, transfected with miR-629 inhibitor plasmids.

A Position 1057-1063 of FOXO3 3'UTR 5' .... AUGUGCGAAGAGUAAAAACCCAA. hsa-miR-629 3' UCAagaggguUgcauUuggGu

Position of mutated FOXO3 3'UTR $\quad 5^{\prime}$... AUGUGCGAAGAGUAACCCGGGAA...

B

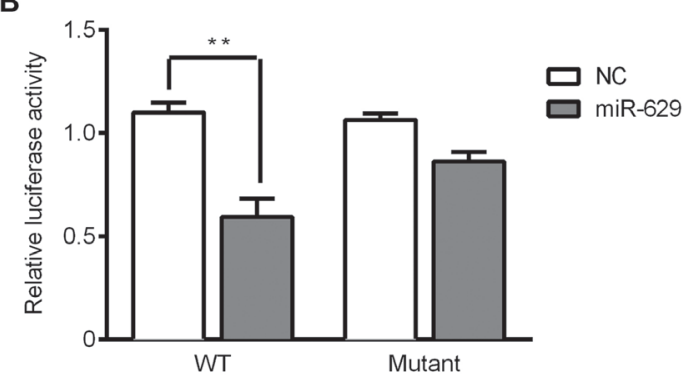

Figure 6. miR-629 target verification via a luciferase reporter assay. (A) TargetScan predicted the binding site (underlined) of miR-629 and the 3'-UTR of FOXO3 mRNA. (B) Luciferase activity following plasmid transfection. ${ }^{* *} \mathrm{P}<0.01$ vs. the negative control group. miR, microRNA; FOXO3, forkhead box O3; UTR, untranslated region; NC, negative control; miR-629 group, miR-629 overexpression plasmid; WT (wild-type), transfected with wild-type FOXO3 3'UTR; MUT (mutant), transfected with mutated FOXO3 3'UTR. 
$\beta$ cells and non-small cell lung cancer cells were also revealed to be influenced by the sirtuin 1/FOXO3 and AMP-activated protein kinase/FOXO3 signaling pathway $(33,34)$. Therefore, the impact of various additional factors, such as transcription factors and epithelial-mesenchymal transition factors, on the expression of FOXO3 and thus the progression of GC should be assessed in the future.

In summary, the current study revealed that miR-629 was upregulated in GC and that the inhibition of miR-629 resulted in an increase in FOXO3 expression. Furthermore, upregulated FOXO3 in SGC-7901 cells transfected with the miR-629 inhibitor may have promoted the expression of Bax and caspase-3, and suppressed the expression of $\mathrm{Bcl}-2$, resulting in the repression of cell proliferation and the promotion of cell apoptosis. In addition, the luciferase reporter assay determined that FOXO3 is a direct target of miR-629. Thus, miR-629 may serve essential roles in cell proliferation and apoptosis in GC. Furthermore the inhibition of miR-629 may be an effective therapy for patients with GC in the future.

\section{Acknowledgements}

Not applicable.

\section{Funding}

No funding received.

\section{Availability of data and materials}

The datasets used and/or analyzed during the current study are available from the corresponding author on reasonable request.

\section{Authors' contributions}

ML drafted the manuscript. ML, YW and XL analyzed the results and data, and collected materials. $\mathrm{ZZ}$ and $\mathrm{LW}$ conducted the experiments. YL conceived of and designed the study, and revised the manuscript. All authors read and approved the final manuscript.

\section{Ethics approval and consent to participate}

The current study was approved by the Ethics Committee of The First Hospital of Shijiazhuang City (Shijiazhuang City, China). All patients provided their written informed consent.

\section{Patient consent for publication}

Not applicable.

\section{Competing interests}

The authors declare that they have no competing interests.

\section{References}

1. Bertuccio P, Chatenoud L, Levi F, Praud D, Ferlay J, Negri E, Malvezzi M and La Vecchia C: Recent patterns in gastric cancer: A global overview. Int J Cancer 125: 666-673, 2009 .
2. Cha Y, He Y, Ouyang K, Xiong H, Li J and Yuan X: MicroRNA-140-5p suppresses cell proliferation and invasion in gastric cancer by targeting WNT1 in the $\mathrm{WNT} / \beta$-catenin signaling pathway. Oncol Lett 16: 6369-6376, 2018.

3. Hamashima C: Current issues and future perspectives of gastric cancer screening. World J Gastroenterol 20: 13767-13774, 2014.

4. He Z, Wang X, Huang C, Gao Y, Yang C, Zeng P and Chen Z: The FENDRR/miR-214-3P/TET2 axis affects cell malignant activity via RASSF1A methylation in gastric cancer. Am J Trans Res 10: 3211-3223, 2018.

5. Yang Y, Shen J, Yan D, Yuan B, Zhang S, Wei J and Du T: Euchromatic histone lysine methyltransferase 1 regulates cancer development in human gastric cancer by regulating E-cadherin. Oncol Lett 15: 9480-9486, 2018.

6. Correa P: Gastric cancer: Overview. Gastroenterol Clin North Am 42: 211-217, 2013

7. Shin VY, Ng EK, Chan VW, Kwong A and Chu KM: A three-miRNA signature as promising non-invasive diagnostic marker for gastric cancer. Mol Cancer 14: 202, 2015

8. Orditura M, Galizia G, Sforza V, Gambardella V, Fabozzi A, Laterza MM, Andreozzi F, Ventriglia J, Savastano B, Mabilia A, et al: Treatment of gastric cancer. World J Gastroenterol 20: 1635-1649, 2014

9. Wu HH, Lin WC and Tsai KW: Advances in molecular biomarkers for gastric cancer: miRNAs as emerging novel cancer markers. Expert Rev Mol Med 16: e1, 2014.

10. Wang LK, Xie XN, Song XH, Su T, Chang XL, Xu M, Liang B and Huang DY: Upregulation of miR-200b inhibits hepatocellular carcinoma cell proliferation and migration by targeting HMGB3 protein. Technol Cancer Res Treat 17: 1533033818806475, 2018.

11. Lan H, Lu H, Wang X and Jin H: MicroRNAs as potential biomarkers in cancer: Opportunities and challenges. Biomed Res Int 2015: 125094, 2015.

12. Van Schooneveld E, Wildiers H, Vergote I, Vermeulen PB, Dirix LY and Van Laere SJ: Dysregulation of microRNAs in breast cancer and their potential role as prognostic and predictive biomarkers in patient management. Breast Cancer Res 17: 21, 2015.

13. Wang Y, Zhang S, Bao H, Mu S, Zhang B, Ma H and Ma S: MicroRNA-365 promotes lung carcinogenesis by downregulating the USP33/SLIT2/ROBO1 signaling pathway. Cancer Cell Int 18: 64, 2018.

14. Liu HS and Xiao HS: MicroRNAs as potential biomarkers for gastric cancer. World J Gastroenterol 20: 12007-12017, 2014.

15. Wang M, Gu H, Qian H, Zhu W, Zhao C, Zhang X, Tao Y, Zhang $\mathrm{L}$ and $\mathrm{Xu}$ W: miR-17-5p/20a are important markers for gastric cancer and murine double minute 2 participates in their functional regulation. Eur J Cancer 49: 2010-2021, 2013.

16. Sun C, Yao X, Jiang Q and Sun X: miR-106b targets DAB2 to promote hepatocellular carcinoma cell proliferation and metastasis. Oncol Lett 16: 3063-3069, 2018.

17. Liu LJ, Yu JJ and Xu XL: MicroRNA-93 inhibits apoptosis and promotes proliferation, invasion and migration of renal cell carcinoma ACHN cells via the TGF- $\beta /$ Smad signaling pathway by targeting RUNX3. Am J Transl Res 9: 3499-3513, 2017.

18. Yan H, Li Q, Wu J, Hu W, Jiang J, Shi L, Yang X, Zhu D, Ji M and Wu C: MiR-629 promotes human pancreatic cancer progression by targeting FOXO3. Cell Death Dis 8: e3154, 2017.

19. Di Vincenzo S, Heijink IH, Noordhoek JA, Cipollina C, Siena L, Bruno A, Ferraro M, Postma DS, Gjomarkaj M and Pace E: SIRT1/FoxO3 axis alteration leads to aberrant immune responses in bronchial epithelial cells. J Cell Mol Med 22: 2272-2282, 2018.

20. Kim SY, Kim HJ, Byeon HK, Kim DH and Kim CH: FOXO3 induces ubiquitylation of AKT through MUL1 regulation. Oncotarget 8: 110474-110489, 2017.

21. Yu S, Yu Y, Zhang W, Yuan W, Zhao N, Li Q, Cui Y, Wang Y, Li W, Sun Y and Liu T: FOXO3a promotes gastric cancer cell migration and invasion through the induction of cathepsin $\mathrm{L}$. Oncotarget 7: 34773-34784, 2016.

22. Park SH, Jang KY, Kim MJ, Yoon S, Jo Y, Kwon SM, Kim KM Kwon KS, Kim CY and Woo HG: Tumor suppressive effect of PARP1 and FOXO3A in gastric cancers and its clinical implications. Oncotarget 6: 44819-44831, 2015.

23. Livak KJ and Schmittgen TD: Analysis of relative gene expression data using real-time quantitative PCR and the 2(-Delta Delta C(T)) method. Methods 25: 402-408, 2001.

24. Xiong H, Wang J, Guan H, Wu J, Xu R, Wang M, Rong X, Huang K, Huang J, Liao Q, et al: SphK1 confers resistance to apoptosis in gastric cancer cells by downregulating Bim via stimulating Akt/FoxO3a signaling. Oncol Rep 32: 1369-1373, 2014. 
25. Ho KK, Myatt SS and Lam EW: Many forks in the path: Cycling with FoxO. Oncogene 27: 2300-2311, 2008

26. Hagenbuchner J, Kuznetsov A, Hermann M, Hausott B, Obexer P and Ausserlechner MJ: FOXO3-induced reactive oxygen species are regulated by BCL2L11 (Bim) and SESN3. J Cell Sci 125 1191-1203, 2012.

27. Essers MA, Weijzen S, de Vries-Smits AM, Saarloos I, de Ruiter ND, Bos JL and Burgering BM: FOXO transcription factor activation by oxidative stress mediated by the small GTPase Ral and JNK. EMBO J 23: 4802-4812, 2004.

28. Zhang X, Tang N, Hadden TJ and Rishi AK: Akt, FoxO and regulation of apoptosis. Biochim Biophys Acta 1813: 1978-1986, 2011.

29. Dall' Acqua A, Sonego M, Pellizzari I, Pellarin I, Canzonieri V, D'Andrea S, Benevol S, Sorio R, Giorda G, Califano D, et al: CDK6 protects epithelial ovarian cancer from platinum-induced death via FOXO3 regulation. EMBO Mol Med 9: 1415-1433, 2017.

30. Yang F, Xu Y, Liu C, Ma C, Zou S, Xu X, Jia J and Liu Z: NF- $\kappa \mathrm{B} / \mathrm{miR}-223-3 \mathrm{p} / \mathrm{ARID} 1 \mathrm{~A}$ axis is involved in Helicobacter pylori CagA-induced gastric carcinogenesis and progression. Cell Death Dis 9: 12, 2018.
31. Xu X, Gao F, Wang J, Tao L, Ye J, Ding L, Ji W and Chen X: MiR-122-5p inhibits cell migration and invasion in gastric cancer by down-regulating DUSP4. Cancer Biol Ther 19: 427-435, 2018.

32. Feng Q, He P and Wang Y: MicroRNA-223-3p regulates cell chemo-sensitivity by targeting $\mathrm{FOXO} 3$ in prostatic cancer. Gene 658: 152-158,2018.

33. Wang N, Zhang J, Qin M, Yi W, Yu S, Chen Y, Guan J and Zhang R: Amelioration of streptozotocin-induced pancreatic $\beta$ cell damage by morin: Involvement of the AMPK-FOXO3-catalase signaling pathway. Int J Mol Med 41: 1409-1418, 2018.

34. Li J, Li P, Chen T, Gao G, Chen X, Du Y, Zhang R, Yang R, Zhao W, Dun S, et al: Expression of microRNA-96 and its potential functions by targeting FOXO3 in non-small cell lung cancer. Tumour Biol 36: 685-692, 2015.

This work is licensed under a Creative Commons Attribution-NonCommercial-NoDerivatives 4.0 International (CC BY-NC-ND 4.0) License. 